\title{
Commentary: Transposition with aortic arch obstruction: The lesions switched?
}

\author{
Shyh-Jye Chen, $\mathrm{MD}, \mathrm{PhD},{ }^{\mathrm{a}}$ and Shu-Chien Huang, $\mathrm{MD}, \mathrm{PhD}^{\mathrm{b}}$
}

\footnotetext{
From the Departments of ${ }^{\mathrm{a}}$ Radiology and Medical Imaging and ${ }^{\mathrm{b}}$ Surgery, National Taiwan University Hospital, National Taiwan University College of Medicine, Taipei, Taiwan.

Disclosures: Authors have nothing to disclose with regard to commercial support.

Received for publication Aug 26, 2019; revisions received Aug 26, 2019; accepted for publication Aug 28, 2019; available ahead of print Oct 25, 2019.

Address for reprints: Shu-Chien Huang, MD, PhD, Department of Surgery, National Taiwan University Hospital, 7 Chung-Shan South Rd, Taipei 100, Taiwan (E-mail: cvshuang@gmail.com).

J Thorac Cardiovasc Surg 2020;159:600-1

$0022-5223 / \$ 36.00$

Copyright (c) 2019 by The American Association for Thoracic Surgery

https://doi.org/10.1016/j.jtcvs.2019.08.110
}

Pulmonary stenosis is the most common reason for reintervention after arterial switch operation (ASO). ${ }^{1}$ Konstantinov and colleagues ${ }^{2}$ from the Royal Children's Hospital enrolled 844 patients who had undergone ASO over the course of more than 3 decades. Among the 83 patients $(9.8 \%)$ who had undergone aortic arch obstruction (AAO) repair in addition to ASO, early mortality was $6.0 \%$ and reintervention was more common than in patients without AAO $(P<.001)$. Follow-up data were available for 74 patients $(95 \%)$. During the follow-up period (median, 13 years), the 3 main reasons for reintervention were right-sided obstruction (15\%), AAO (5.4\%), and neoaortic insufficiency $(6.8 \%)$. This study is one of the largest series AAO.

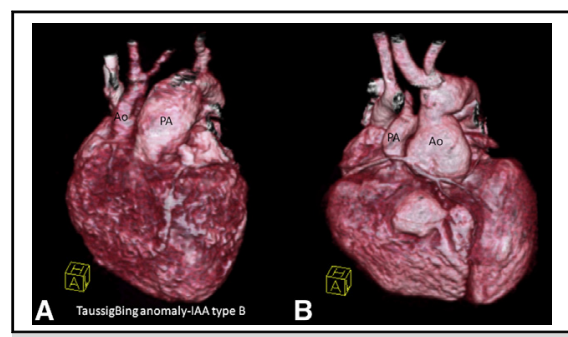

A small aortic annulus (A) became a small pulmonary annulus (B) following arterial switch.

\section{Central Message}

Arterial switch operations in patients with aortic arch obstruction require careful consideration for neopulmonary stenosis, residual aortic arch obstruction, and neoaortic regurgitation.

See Article page 592.

addressing the results in the challenging scenario of patients with transposition of the great arteries in combination with

The need for reintervention can be explained by anatomic factors. Patients with AAO tend to have multileveled obstruction, including subaortic stenosis and smaller aortic

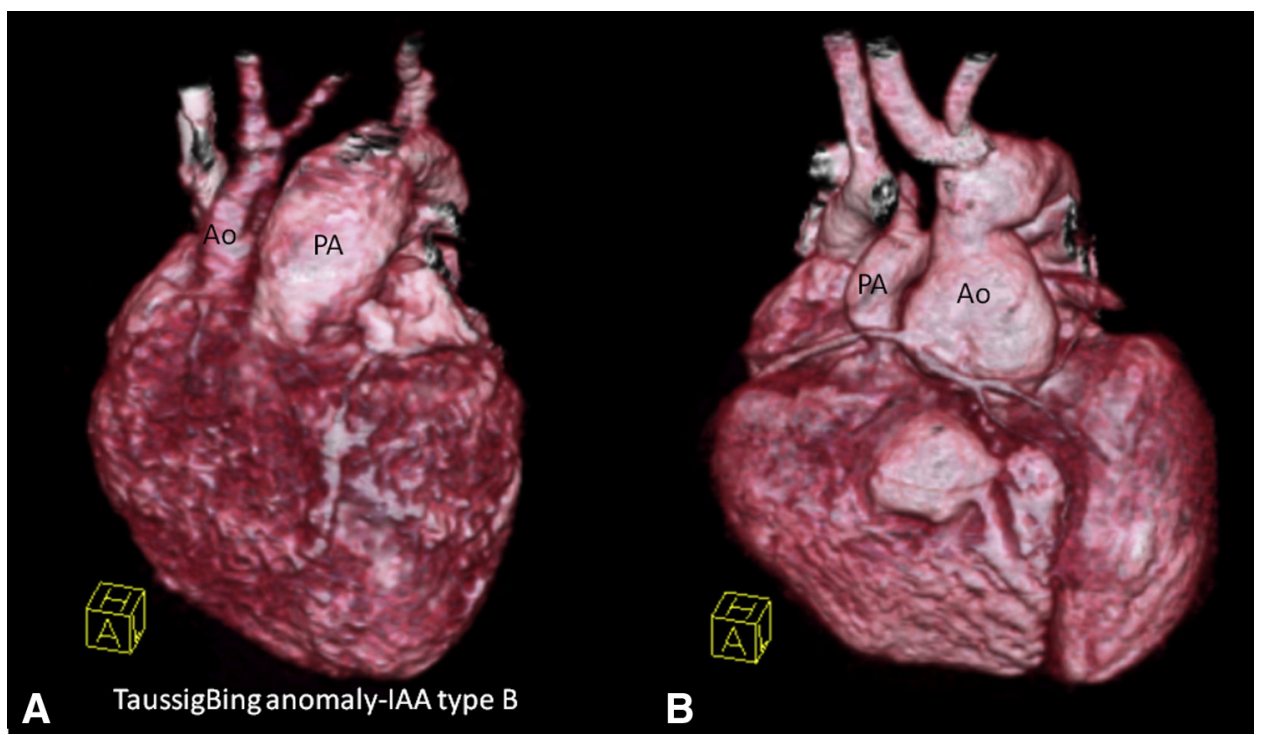

FIGURE 1. Preoperative (A) and postoperative (B) computed tomography images. A 3-dimensional computed tomography scan of Taussig-Bing anomaly and type B interrupted aortic arch. The aortic annulus was small (4 mm). After an arterial switch operation, the small neopulmonary annulus and the right coronary artery crossing the right ventricular outflow tract made the reintervention on the right-sided obstruction inevitable. We used an atrioventricular groove patch for pulmonary valve stenosis in this case. Ao, Aorta; $P A$, pulmonary artery. 
valve annulus, which were switched to neopulmonary outflow, resulting in right-sided obstruction. The large pulmonary valve and the transferred coronary buttons create a larger neoaortic valve, with the potential to lead to regurgitation at later follow-up. (Figure 1).

In this study, patients with the more severe form of AAO (ie, interrupted aortic arch) were at greater risk (hazard ratio, 3.1) of requiring right-sided reintervention. Therefore, we speculate that a greater size discrepancy between the native aorta and pulmonary artery increases the likelihood of requiring intervention. However, the necessary detailed initial anatomic data (eg, size of the aorta or pulmonary valve) could not be obtained in this retrospective study. In patients with interrupted aortic arches and small aortic annuli, smaller preoperative left ventricular outflow structures predict the need for reoperation. ${ }^{3}$ The Yasui operation or a Norwood-type operation may be required to bypass the left ventricular outflow obstruction. ${ }^{4}$ Nevertheless, the answer to the question of how small is too small for switching in patients with a transposition complex and AAO remains unclear.

The reason for reintervention was right-sided obstruction in 11 of 74 surviving patients (15\%), including the branch pulmonary artery $(n=6)$, main pulmonary artery $(n=6)$, neopulmonary valve $(\mathrm{n}=5)$, and subvalvular level $(n=6)$. These results suggest that all levels of obstruction should be carefully addressed in the initial repair, which include resection of subaortic stenosis and proper reconstruction of the neopulmonary artery. ${ }^{5}$
The authors currently perform direct end-to-side connect to anastomosis to correct aortic arch obstruction. This technique is associated with a very low reintervention rate. If the ascending aorta diameter is too small, using patch enlargement of the transverse aortic arch and ascending aorta to control for the important diameter mismatch between the aorta and the pulmonary root will be helpful to prevent supravalvular aortic stenosis.

In summary, this experience in Australia demonstrates excellent long-term survival for the group of patients. Late reintervention is highly related to the initial small aorta size and the size discrepancy between the aorta and pulmonary artery. Although some reintervention seems inevitable, the surgical technique should accommodate patient anatomy and relieve multilevel obstruction of both systemic and pulmonary outflow.

\section{References}

1. Raju V, Burkhart HM, Durham LA III, Eidem BW, Phillips SD, Li Z, et al. Reoperation after arterial switch: a 27-year experience. Ann Thorac Surg. 2013;95: 2105-12.

2. Fricke TA, Donaldson S, Schneider JR, Menahem S, d'Udekem Y, Brizard CP Outcomes of the arterial switch operation in patients with aortic arch obstruction. J Thorac Cardiovasc Surg. 2020;159:592-9.

3. Riggs KW, Tweddell JS. How small is too small? Decision-making and management of the small aortic root in the setting of interrupted aortic arch. Semin Thorac Cardiovasc Surg. 2019;22:21-6.

4. Alsoufi B, Schlosser B, McCracken C, Sachdeva R, Kogon B, Border W, et al. Selective management strategy of interrupted aortic arch mitigates left ventricular outflow tract obstruction risk. J Thorac Cardiovasc Surg. 2016; 151:412-20.

5. Lacour-Gayet F. Arterial switch operation with ventricular septal defect repair and aortic arch reconstruction. Semin Thorac Cardiovasc Surg. 2007;19:245-8. 